\title{
HEMATIMETRIC INDICES AS BIOMARKERS OF BACTERIAL INFECTION IN SYSTEMIC LUPUS ERYTHEMATOSUS
}

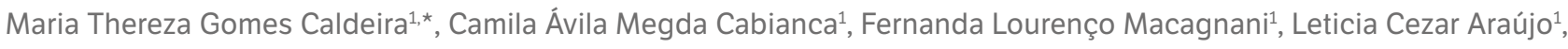 \\ Renê Donizeti Ribeiro de Oliveira ${ }^{1}$ \\ 1.Universidade de São Paulo, Ribeirão Preto (SP), Brazil. \\ *Corresponding author: emailnumero9@gmail.com
}

\section{BACKGROUND}

Systemic lupus erythematosus (SLE) is a chronic disorder associated with a substantial hospitalization risk. Distinction between infection and flare in patients with SLE is a challenge in clinical practice. Hematimetric indices, such as neutrophil-to-lymphocyte ratio (NLR), platelet-to-lymphocyte ratio (PLR) and systemic immune inflammatory index (SII) (neutrophil $\times$ platelet / lymphocyte) are considered and used as biomarkers of immune system activation and response. We aimed to investigate the performance of these indices in SLE patients under two different conditions: during the course of systemic bacterial infection and after recovery.

\section{METHODS}

We present a cross-sectional, observational study with data collected from clinical records of patients aged between 18 and 65 years, with a previous diagnosis of SLE, admitted to a tertiary hospital for intravenous treatment of bacterial infection. Data were collected from January 1, 2018 to January 1, 2019, with a number of 23 patients allocated to the study. Patients were evaluated at two times, during the acute stage of infection and after treatment. The data collected were: age, gender, organ initially affected by the infection, immunosuppressant being used to treat the underlying disease and hematimetric indices at admission and after treatment at the subsequent outpatient clinical evaluation.

\section{RESULTS}

The mean age was 26 years and $96 \%$ of the patients were female. Approximately $86 \%$ of the patients evaluated were using immunosuppressants (30\% in pulse therapy with cyclophosphamide, $26 \%$ with azathioprine, $17 \%$ with methotrexate, $9 \%$ with mycophenolate mofetil, $4 \%$ with immunosuppressive dose of a glucocorticoid). The most common location of infections was pulmonary (26\%), followed by skin (22\%) and genitourinary tract (17\%). Only the mean of PLR showed statistical significance when comparing the admission and the post-treatment evaluations ( $443.0 \pm 400.9$ vs. $254.8 \pm 136.3 ; p=0.03$ ).

\section{CONCLUSION}

There are few descriptions of the use of PLR, NLR and SII calculations in patients with SLE infected and hospitalized. One report found that NLR together with CRP levels increased sensitivity and negative predictive value as an attempt to differentiate the non-viral infectious picture from disease activity in SLE. We could not find this association, but in our patients, PLR was able to differentiate patients in two distinct phases in the course of SLE, with and without systemic bacterial infection. Although this study was conducted with a small number of patients, we found that PLR may become a biomarker of systemic infection in patients with SLE regularly treated with immunosuppressive drugs.

\section{KEYWORDS}

Systemic lupus erythematosus, Infection, Hospitalization. 